\title{
Following Dynamic Processes by X-ray Tomographic Microscopy with Sub-second Temporal Resolution
}

\author{
R. Mokso ${ }^{\mathrm{a}}$, F. Marone ${ }^{\mathrm{a}}$, D. Haberthür ${ }^{\mathrm{b}}$, J.C. Schittny ${ }^{\mathrm{b}}$, G. Mikuljan ${ }^{\mathrm{a}}$, A. Isenegger ${ }^{\mathrm{a}}$, \\ and M. Stampanoni ${ }^{\mathrm{a}, \mathrm{c}}$ \\ ${ }^{a}$ Swiss Light Source, Paul Scherrer Institut, 5232 Villigen, Switzerland \\ ${ }^{b}$ Institute of Anatomy, University of Bern, Balzerstrasse 2, 3012 Bern, Switzerland \\ ${ }^{c}$ Institute for Biomedical Engineering, University and ETH Zurich, Gloriastrasse 35, 8092 Zurich, Switzerland
}

\begin{abstract}
Several non-destructive imaging techniques offer the possibility to observe rapid phenomena in real time, yet most of these techniques fail when it comes to bulky samples and micrometer precision in three dimensions. Therefore there is clearly a need to develop approaches that address such conditions. We identified the large potential that lies in synchrotron-based x-rays as a probe and developed a direct-space tomographic instrument suitable to provide sub-second temporal resolution with several-micrometers spatial resolution. Selected applications from the field of biology and material science are shown in order to demonstrate the unique capabilities in generating three-dimensional images with very high quality making image segmentation and analysis possible for samples that could, until now, only be studied in two dimensions due to the occurrence of rapid structural changes.
\end{abstract}

Keywords: Fast tomography, imaging, in-situ, in-vivo, foams

PACS: $0.7 .85 . \mathrm{Qe}, 07.85 . \mathrm{Tt}$, 81.70.Tx, 47.57.Bc

\section{INTRODUCTION}

The interest to study three-dimensional systems that exhibit fast evolution is one of the major motivations for the efforts put into the improvement of the temporal resolution in x-ray microtomography. A tomographic dataset is composed of individual radiographic projections of the sample at equidistant angular positions between 0 and $\pi$. The necessary condition for a correct tomographic reconstruction is that the sample remains unchanged until all the projections are acquired. If this condition is not satisfied, motion artifacts arise, preventing the quantitative analysis (segmentation, for instance) of the images. There are two ways to satisfy this condition. The first is to ensure the stability of the sample, and the second is to acquire the tomographic dataset faster. The first requirement may in some cases impair the study of interesting dynamical systems like liquid and aluminium foams, sintering of particles, and breathing and heart cycle of small animals. The access of relevant parameters in complex, threedimensional samples undergoing rapid structural modifications is hence only possible if these changes occur at rates matched to the temporal resolution of the probing process.

Ultrafast radiography has been demonstrated at various beamlines [1-4], and a number of exciting applications have been successfully addressed [5]. Nevertheless, the extreme requirements on mechanics, synchronization, and detectors prevent a straightforward transition from 2D towards 3D ultrafast imaging. With the following results we demonstrate that this important step can now be achieved.

\section{METHODS}

There are three principal factors that determine the feasibility of ultrafast tomography: (i) the brilliance of the $\mathrm{x}$ ray source, (ii) the detector speed and sensitivity, and (iii) the synchronization of acquisition. In the following we will address each of these elements.

The x-ray source of the TOMCAT beamline [6] of the Swiss Light Source is a 2.9-T superbending magnet. The photon flux density at the sample position is $5 \times 10^{11}$ photons $/ \mathrm{s} / \mathrm{mm}^{2}$ for monochromatic photons of $20 \mathrm{keV}$, which

The 10th International Conference on X-ray Microscopy

AIP Conf. Proc. 1365, 38-41 (2011); doi: 10.1063/1.3625299

(C) 2011 American Institute of Physics 978-0-7354-0925-5/\$30.00 
was used for the current study. The multilayer monochromator $(\mathrm{Ru} / \mathrm{C}$ for energies of $8-21 \mathrm{keV}$ or W/Si for higher energies up to $45 \mathrm{keV}$ ) provides a monochromatic beam with a bandwidth of $2 \%$ and reduces the photon flux by approximately two orders of magnitude. The measurements covered in this paper were performed either with monochromatic $\mathrm{x}$-rays at $20 \mathrm{keV}$ or polychromatic radiation from the superbending magnet.

For high-spatial-resolution direct space imaging with frame rates above $100 \mathrm{~Hz}$, the choice of the detection system is very limited. Although the development of new pixel detectors is approaching smaller pixel sizes and higher stopping power for hard $\mathrm{x}$-rays, these systems are not yet mature for tomographic microscopy. Indirect detection in the form of a visible light camera coupled to a light conversion screen (scintillator) is currently the most suitable configuration. Despite technological advances such as the operation in frame transfer mode, CCD-based detectors with a sufficient number of pixels ( $>2$ Mpixels) have readout times of a few hundred milliseconds at best, which is comparable or longer than the exposure itself. Taking this into account, the choice of CMOS cameras with more than three orders of magnitude shorter readout seems obvious. In this study, we use the new pco.Dimax CMOS camera, coupled to a $250-\mu \mathrm{m}$-thick YAG:Ce scintillator. The fluorescence curve of this scintillator (peaking around the value of $550 \mathrm{~nm}$ ) was well matched to the maximum quantum efficiency of the camera $(50 \%$ at $600 \mathrm{~nm})$. The image formed by the scintillator was directly $1: 1$ projected to the detector sensor using tandem optics. No microscope objective has been used in the current configuration, meaning that the effective spatial sampling of the image on the detector was $11 \mu \mathrm{m}$, as given by the physical pixel size of the camera chip. Nearly 12-bit dynamic range operation of the camera was achieved as the shot noise (including dark current and readout noise) was observed to be at a value of about 300 out of 4096 gray levels.

We chose to synchronize the tomographic acquisition process by allowing the rotation stage with the sample mounted on it to rotate continuously at an adequate speed calculated from the frame rate of the detector. The airbearing rotary stage (currently a limiting factor) can perform up to $360^{\circ} / \mathrm{s}$, allowing the acquisition of up to two tomographic data set within 1 second even though the photon flux would allow a 10-15 times faster acquisition speed at good quality. In this continuous mode, the camera is triggered automatically to acquire one or more complete sets of projections.

\section{EXPERIMENTAL RESULTS}

We demonstrate the capabilities of the ultrafast tomography setup on four selected samples, each of them scanned under different conditions. The first sample is a trabecular bovine bone with well-known 3D structure. At $20 \mathrm{keV}$ the absorption contrast is optimal, making this sample suited for the assessment of the image quality when optimizing the acquisition protocol.
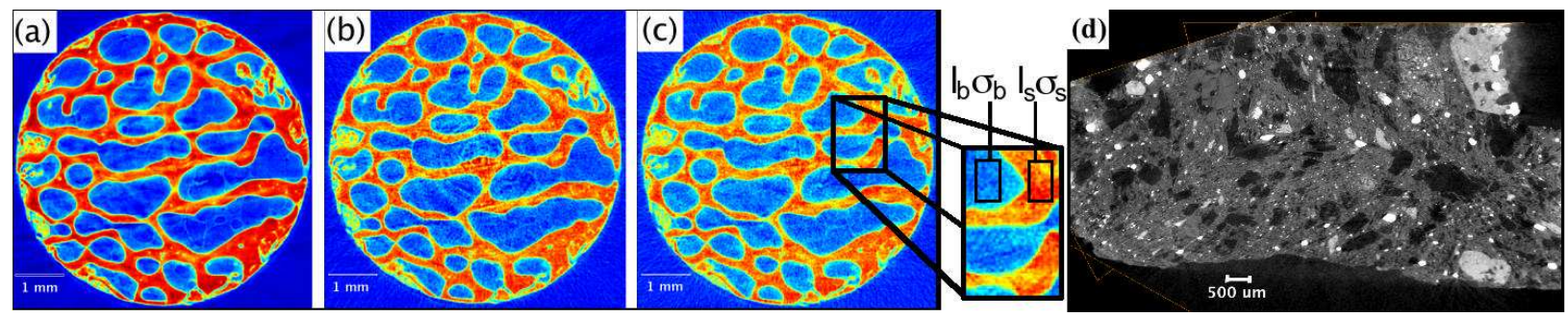

FIGURE 1. (a), (b), and (c) are tomographic slices of a bovine trabecular bone biopsy obtained with 20-keV monochromatic beam and three different acquisition protocols. Image (a) corresponds to optimal illumination conditions with exposure time $t_{\text {exp }}=$

$6 \mathrm{~ms}$, number of projections $\mathrm{N}_{\text {tomo }}=600$, and total scan time $\mathrm{t}_{\text {scan }}=3.7 \mathrm{~s}$. Image (b) is for moderate sampling with $t_{\exp }=1.1 \mathrm{~ms}$,

$\mathrm{N}_{\text {tomo }}=600$, and $\mathrm{t}_{\mathrm{scan}}=0.67 \mathrm{~s}$. Image (c) is for minimal sampling, with $\mathrm{t}_{\mathrm{exp}}=1.65 \mathrm{~ms}, \mathrm{~N}_{\text {tomo }}=300$, and $\mathrm{t}_{\mathrm{scan}}=0.5 \mathrm{~s}$. In (d) the

orthoslices represent a lava sample scanned in polychromatic (white beam) configuration with $\mathrm{t}_{\text {exp }}=700 \mu \mathrm{s}, \mathrm{N}_{\text {tomo }}=1000$, and $\mathrm{t}_{\mathrm{scan}}=0.7 \mathrm{~s}$.

For an extensive systematic study of image quality dependence on the acquisition parameters, the trabecular bone sample was imaged with monochromatic x-rays using the double-crystal multilayer monochromator. The sample was placed very close (about $1 \mathrm{~mm}$ ) to the scintillator screen to minimize contamination of the images by the phase contrast component. All the reconstructions in Fig. 1 were done with the Fourier-based tomographic reconstruction method that utilizes prolate functions for interpolation in Fourier space [7]. The signal-to-noise ratio (SNR) and the spatial resolution have been used as figures of merit in the evaluation. We use the definition given by $\mathrm{SNR}=\left(<\mathrm{I}_{\mathrm{S}}>-\right.$ 
$\left.<\mathrm{I}_{\mathrm{b}}>\right) /\left(\sigma_{\mathrm{s}}^{2}+\sigma_{\mathrm{b}}^{2}\right)^{1 / 2}$, where I and $\sigma$ are the mean pixel value and the corresponding standard deviation of the signal and background region, respectively, as defined on the inset in Fig. 1. This resulted in the following values: (a) SNR=22, (b) $\mathrm{SNR}=13.5$, and (c) $\mathrm{SNR}=11.5$. Note that by improving the temporal resolution by a factor of 7.5 from scan (a) to scan (c), the SNR gets poorer by only a factor of 2. The number of projections $\mathrm{N}_{\text {tomo }}$ required for the optimal reconstruction of a tomographic set is related to the number of pixels $\mathrm{N}_{\text {pix }}$ in the direction perpendicular to the rotation axis as $\mathrm{N}_{\text {opt }}=\mathrm{N}_{\text {pix }} \cdot \pi / 2$. The sample region in our case is composed of $\mathrm{N}_{\text {pix }}=1200$ pixels. Decreasing the sampling to about $\mathrm{N}_{\text {opt }} / 4$ does not affect significantly the quality of reconstructed data. The spatial resolution was evaluated with two methods: the first based on power spectrum analysis [8] and the second by fitting line profiles. A value of 2.5 pixels was found for all scans, meaning that mainly the SNR is significantly affected by this reduction of the number of projections or exposure time.

When operating with exposure times as short as one millisecond, the system becomes particular sensitive to high-frequency vibrations in the monochromator, installed 18 meters upstream from the sample. This results in timedependent background alterations on the individual projections, and this in turn may deteriorate the quality of the tomographic reconstruction. Sophisticated background correction routines may bring improvement, or the issue can be obviously overcome by omitting the monochromator and using polychromatic beam. In this setup an absorbing lava (Sibayak volcano, Sumatra) sample has been scanned, see Fig. 1(d). The tomograms of this multi-component sample are of high quality. The total scanning time was $0.7 \mathrm{~s}$. The exposure time of $700 \mu \mathrm{s}$ was sufficient to exploit the full dynamic range of the 12-bit CMOS chip using only $5 \%$ of the white beam power spectrum filtered by a carbon filter.

A wide range of applications will benefit from the recent achievements in ultrafast microtomography. We selected here two pilot studies, one biological and one material science related.

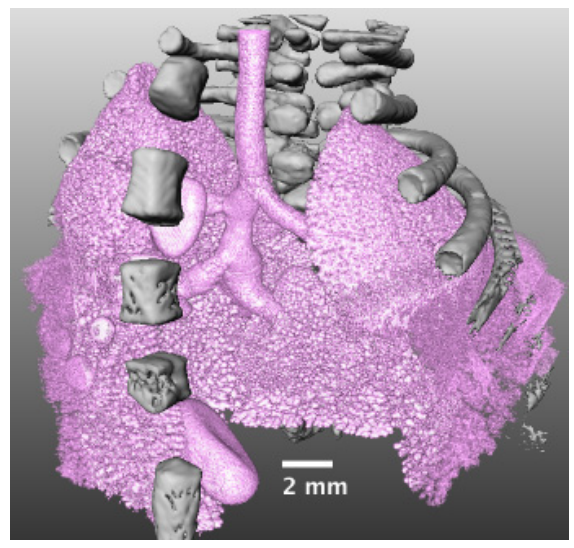

FIGURE 2. Volume rendering of the breast area of a 5-day-old rat. Three consecutive tomographic scans were stitched in the vertical direction to obtain the complete volume. Each tomogram was acquired in $0.5 \mathrm{~s}$, with exposure time $\mathrm{t}_{\mathrm{exp}}=1.1 \mathrm{~ms}$, number of projections $\mathrm{N}_{\text {tomo }}=600$, total scan time $\mathrm{t}_{\text {scan }}=0.7 \mathrm{~s}$, and pixel size of $11 \mu \mathrm{m}$.

Figure 2 depicts volume rendering of the complete lungs of a 5-day-old rat. The animal was sacrified before the experiment and without any further preparation inserted into the monochromatic x-ray beam. The beam energy of 20 $\mathrm{keV}$ provides sufficient transmission also in the presence of the supporting skeleton, and at the same time the phaseenhanced images are suitable to show in detail the soft lung tissue. The distance between the sample and detector was chosen carefully by ensuring that the first Fresnel fringe occurring due to interference of the partial coherent $\mathrm{x}$ rays is not exceeding the size of one pixel in the image. In such a way, edge-enhanced phase-contrast images provide high-resolution information on low-contrast samples. Due to the smaller vertical size of the probing beam, the complete 3D image is composed of 3 individual tomograms stitched in the vertical direction. The largest diameter of the lung tissue was about $20 \mathrm{~mm}$. This pilot study supports the feasibility of in vivo experiments following the development and physiological altering of the lung structure. This can shed light on some remaining questions previously addressed with experiments using embedded or fixed samples [9].

The second example concerns the study of the coarsening of liquid foam. Beer foam was prepared ex situ with a liquid fraction of $5-15 \%$ and placed on the rotation table in the x-ray beam. In contrast to the previous examples, these liquid foams are rapidly evolving complex systems and can not be studied without adequately matched speed of data acquisition. Similar to the soft tissue case, the phase shift of the x-rays produces the image contrast. The films dividing the bubbles are too thin to be visualized; however, the plateau borders are well defined and are 
sufficient to recognize and label individual bubbles [10]. The first 3D studies with good statistics had been performed in earlier works $[10,11]$ using similar types of liquid foams, but with additional surfactants to regulate gas exchange between bubbles and to slow down the foam coarsening. As stated in [10], the investigation of the evolution of the early stages of such foams has not been possible in 3D so far because of the rapid dynamics. We show (Fig. 3) that 3D reconstructions free of motion artifacts are now obtained; this opens new possibilities to verify the theoretical models [12] developed but until now only supported by 2D experimental data. The three images correspond to different stages of foam evolution all within the first minute of the foam aging process. These wet foam tomograms serve in addition to demonstrate that in spite of the $360^{\circ} / \mathrm{s}$ rotation speed, the centrifugal forces do not cause instability of the liquid sample.
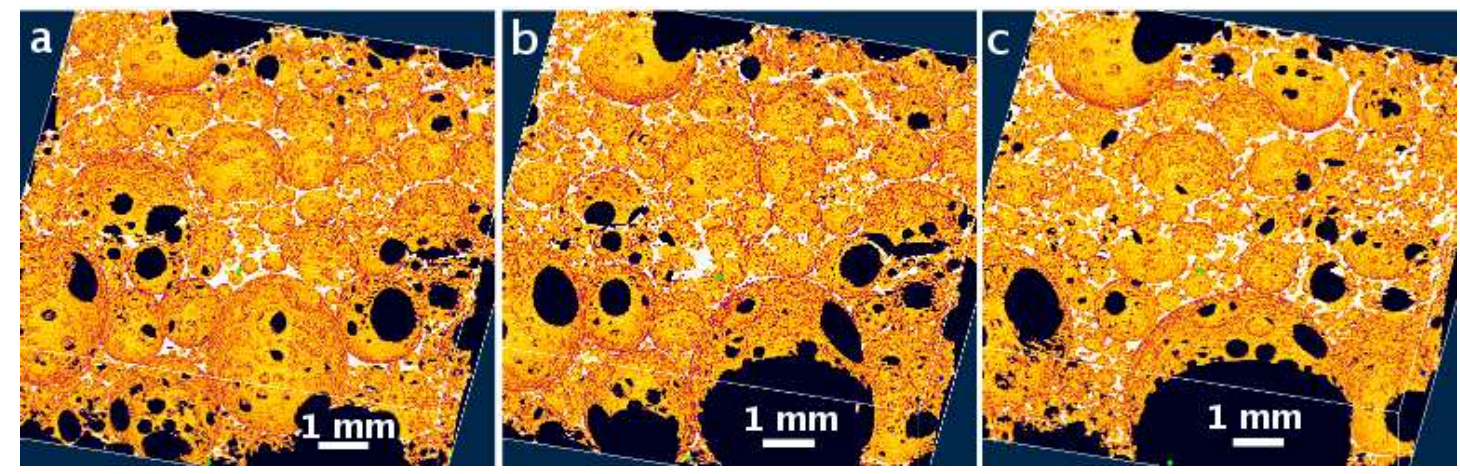

FIGURE 3. The 3D dynamics of a rapidly evolving beer foam as visualized by volume rendering at different time instances during natural coarsening: (a) $t_{0}$, (b) $t_{0}+3 \mathrm{~s}$, and (c) $t_{0}+7 \mathrm{~s}$. Total scan time for a set of tomographic images was $0.5 \mathrm{~s}$, and exposure time for each projection $\mathrm{t}_{\mathrm{exp}}=2 \mathrm{~ms}$.

\section{CONCLUSIONS AND OUTLOOK}

The TOMCAT beamline provides sufficient brilliance to enable exposure times as small as $1 \mathrm{~ms}$ or less for individual projections with monochromatic beam (highest flux for $15-21 \mathrm{keV}$ ), while the tomographic reconstructions remain of high quality. Omitting the multilayer monochromator, the flux increases by two orders of magnitude with corresponding shortening of the exposure time. In the current study the temporal resolution of the tomographic acquisition was limited to $0.5 \mathrm{~s}$ due to the maximum speed of the rotation stage.

One goal of the real-time tomography project at TOMCAT is the visualization of the acquired 3D volume or its part at rates comparable to the total acquisition times. For this purpose, fast tomographic reconstruction procedures are being developed [7] with the potential to allow 3D visualization synchronized with the acquisition. The reconstruction algorithm based on the Fourier transform method has the potential to meet the stringent requirements provided the processing of the data is fully parallelized.

The experimental results presented here clearly indicate that this technique has reached the maturity to address in situ studies of different kinds as well as to establish in vivo imaging of small animals in 3D using x-ray attenuation or phase shift as contrast mechanism.

\section{REFERENCES}

1. A. Rack, F. Garcia-Moreno, T. Baumbach and J. Banhart, J. Synchrotron Radiat. 16, 432 (2009).

2. M. Di Michiel et al., Rev. Sci. Instrum. 76, 043702 (2005).

3. K. Uesugi, T. Sera and N. Yagi, J. Synchrotron Radiat. 13, 403 (2006).

4. K.-S. Im et al., Appl. Phys. Lett. 90, 091919 (2007).

5. N. Babcsan, F.G. Moreno, J. Banhart, Colloid Surface A 309, 254 (2007).

6. M. Stampanoni et al., Proc. SPIE 6318,, 63180C (2006).

7. F. Marone, C. Hintermueller, R. Geus, and M. Stampanoni, IEEE Nuclear Science Symp. Conf. Proc., 2009 , p. 5469.

8. P. Modregger, D. Lubbert, P. Schafer, and R. Kohler, Physica Status Solidi A 204, 2746 (2007).

9. J.C. Schittny, S.I. Mund, and M. Stampanoni, Am. J. Physiol. 294, L246 (2008).

10. J. Lambert et al., Phys. Rev. Lett. 99, 058304 (2007).

11. J. Lambert et al., Phys. Rev. Lett. 104, 248304 (2010).

12. C. Raufaste, S.J. Cox, P. Marmottant, and F. Graner, Phys. Rev. E 81, 031404 (2010). 